Automotive and

Traffic Systems Technology

18. Internationaler Kongress /

$18^{\text {th }}$ International Congress
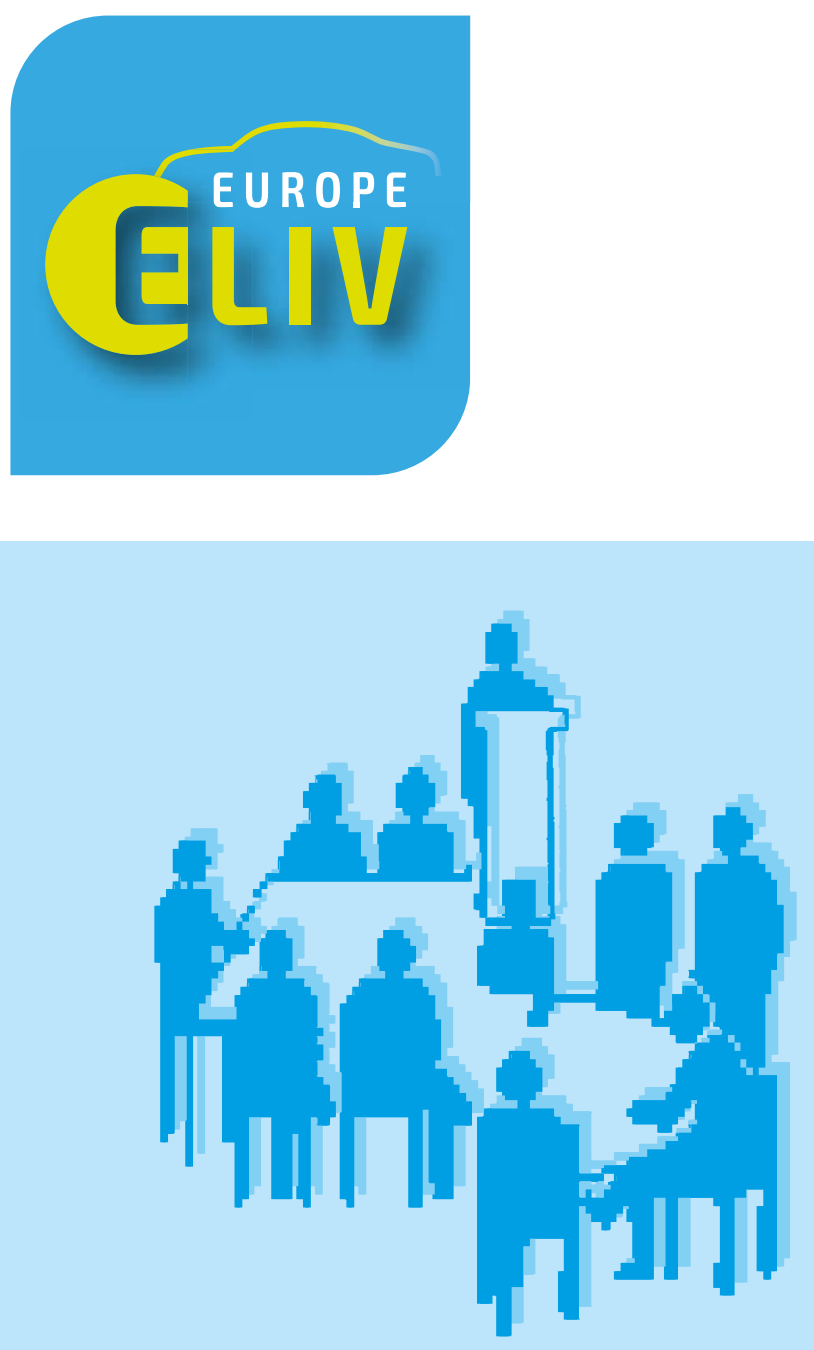

VDI-Berichte 2299 
VDI-BERICHTE

Herausgeber: VDI Wissensforum GmbH 
https://doi.org/10.51202/9783181022993-1

Generiert durch IP '172.22.53.54', am 26.04.2023, 10:38:12.

Das Erstellen und Weitergeben von Kopien dieses PDFs ist nicht zulässig. 


\section{Automotive and}

VDI Traffic Systems Technology

18. Internationaler Kongress / $18^{\text {th }}$ Intermational Congress

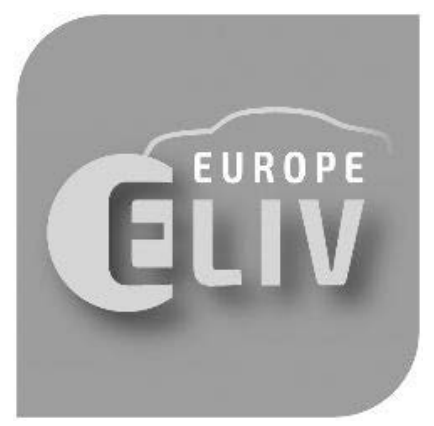

Bonn, 18. and 19. October 2017

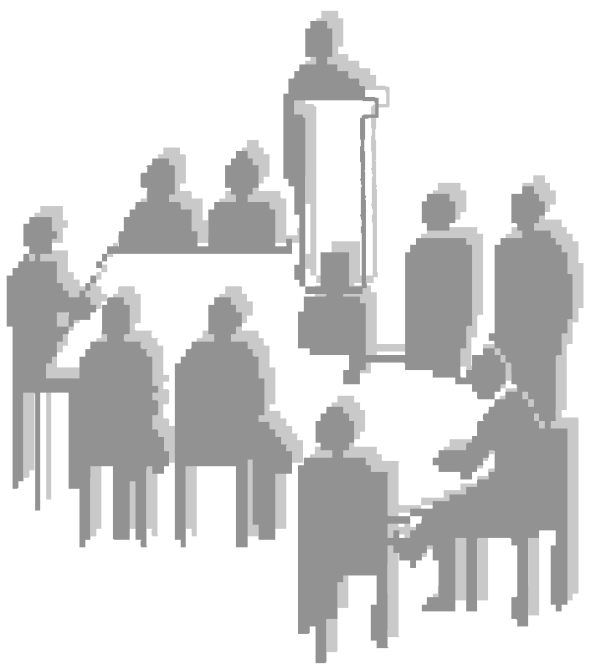

VDI-Berichte 2299 
Bibliographische Information der Deutschen Nationalbibliothek

Die Deutsche Nationalbibliothek verzeichnet diese Publikation in der Deutschen Nationalbibliographie; detaillierte bibliographische Daten sind im Internet unter http://dnb. ddb.de abrufbar.

\section{Bibliographic information published by the Deutsche Nationalbibliothek} (German National Library)

The Deutsche Nationalbibliothek lists this publication in the Deutsche Nationalbibliographie

(German National Bibliography); detailed bibliographic data is available via Internet at http://dnb.ddb.de.

\section{(C) VDI Verlag GmbH · Düsseldorf 2017}

Alle Rechte vorbehalten, auch das des Nachdruckes, der Wiedergabe (Photokopie, Mikrokopie), der Speicherung in Datenverarbeitungsanlagen und der Übersetzung, auszugsweise oder vollständig.

Der VDI-Bericht, der die Vorträge der Tagung enthält, erscheint als nichtredigierter Manuskriptdruck. Die einzelnen Beiträge geben die auf persönlichen Erkenntnissen beruhenden Ansichten und Erfahrungen der jeweiligen Vortragenden bzw. Autoren wieder.

Printed in Germany.

ISSN 0083-5560

ISBN 978-3-18-092299-7 


\section{Hochautomatisiertes Fahren / Highly Automated Driving}

K. Büttner

R. Huisman,

T. Kabos,

M. Beenakkers,

A. -J. van Eck

S. Waschul,

B. Iske,

C. Raksch,

T. Führer

F. Netter,

P. Elspas

U. Muller,

L. D. Jackel,

J. Langenwalter

F. Fitzek,

U. Seger,

D. Lewis,

D. Amey,

E. Dagan

N. Beringer,

O. Koska
BMW 2021: Auf dem Weg

zum automatisierten Fahren

Steps towards highly

automated driving

Hochautomatisiertes Fahren

(HAF) und Parken (HAP) -

Trends und Heraus-

forderungen

Künstliche Intelligenz (KI) für Artificial Intelligence for

Fahrzeuge-Anwendungen, cars-Applications,

Technologien und Herausforderungen

Teaching a car to drive -

Automating the extraction

of domain knowledge by

observing human drivers

Industrieübergreifende

Kooperation zur

Standardisierung von

Kameratechnologien

Erweiterung der Sensor-

reichweite für Hochauto-

matisiertes Fahren: Ein

neuer Ansatz zur

Umfeldmodellierung automated driving: a new approach for environment modelling
BMW 2021: the road

towards Autonomous

Driving

Steps towards highly

7 automated driving
(HAD) and parking

(HAP) - Trends and

challenges

technologies and

challenges

Teaching a car to drive -

Automating the extraction of domain knowledge by observing human drivers

Cross-industry cooperation for camera technology standardization

Extending the sensor 
T. Schaller, W. Uhler, J. Schrepfer

M. Schilling

T. Wilmer, T. Kopfstedt

L. Mesow, F. Knabl
Proposal zur

Standardisierung von

Sensor Interfaces

zu einer Fusionseinheit

Sensor- und Funktions-

schnittstellen in der

Offenen Fusionsplattform (OFP)

Linux in sicherheitskritischen Systemen für zukünftige ADAS und halbautomatisierte Fahrfunktionen

AADC - AUDI Autonomous

Driving Cars: Eine offene Plattform zur Vorentwicklung und Demonstration von automatischen Fahrfunktionen auf Basis von Modellfahrzeugen
Proposal for

19

standardization of sensor interfaces to a fusion unit

Open fusion platform (OFP) - Sensor and functional interfaces systems for future ADAS and semi-automated driving functionality AADC-Audi autonomous driving cars: An open platform for predevelopment and demonstration of automatic driving functions based on model cars

\section{Test - Hochautomatisiertes Fahren / Testing Highly Automated Driving}
R. Belke,
G. Kiffe

FEP - Functional

FEP - Functional

Engineering Platform - Die

engineering platform -

Entwicklungsplattform der FEP solutions enabling AUDI AG für Automatisiertes Fahren the way to automated driving

M. Herrmann,

Echtzeitfähige Sensor-

Real-time-capable 29

D. Dörr modelle für den virtuellen sensor models for virtual Fahrversuch: Klassifikation und Anwendung in test driving: Classification and application in Entwicklung und development and Absicherung automatisierter Fahrfunktionen validation of automated driving functions 


$\begin{array}{ll}\text { H. Abdellatif, } & \text { Virtuelle Homologation von } \\ \text { B. Schick } & \text { softwareintensiven, } \\ & \text { sicherheitsrelevanten } \\ & \text { Systemen: vom ESP bis } \\ & \text { zum Automatisieren Fahren }\end{array}$

Virtual homologation of software intensive safety system: From ESP to autonomous driving

\section{Smart \& Connected Vehicle}
U. Beher,
T. Weyrath

M. Ruf,

J. Lützner

C. Voigt,

J. Plechinger

F. Salzmann,

B. Zerche,

B. Bäker

H. Baumgärtel,

J. Bungalski,

S. Gontscharov,

B. Kanning
IT-Backends für

Automatisiertes Fahren und Kooperative Assistenz

Konnektivität - DIE Grundvoraussetzung für Effizienzsteigerungen in Nutzfahrzeugen

5G Automotive Association als Wegbereiter der digitalen Transformation in der Automotive Industrie

Komponentenbasierte

Programmstruktur zur

Datenvorverarbeitung in vernetzten Fahrzeugen

Tastsinn des Fahrzeugs:

Smarte Sensoren zur

Vibrationsanalyse
IT-Backend for automated 33 driving and cooperative ADAS

Connectivity as THE 35 enabler for efficiency Improvements in commercial vehicles

5G Automotive association - Pioneering the transformation in the automotive industry

Component-based framework for data preprocessing within connected car architectures

Sense of touch for vehicles: Smart sensors for vibration analysis 

S. Max,
Umfeld Sensorik für alle -
Environment sensor for
R. Koch, Serienentwicklung von everybody: serial
T. Büschenfeld, fahrenden Messstationen development of the
P. Baumann,
T. Ruchatz, driving measurement station
S. Ortmann,
A. Titze
T. Häberlein, Rahmenbedingungen für die
Framework conditions for
A. Unger, Implementierung von the implementation of
B. Bäker, backend-basierten backend-based functions
O. Manicke
Funktionen im Kontext von in the context of Connected Vehicles connected vehicles

43

\section{Offboard Ecosysteme / Offboard Ecosystems}
A. Reich, Zugang zu Fahrzeugdaten
Access to vehicle data
N. Krämer,
R. Lenninger
P. Ott, Deep end-to-end Learning
Deep end-to-end learning
C. Speck, im Automotivebereich in automotive
G. Matenaer
J. Kirschbaum
Das digitale Abbild der
Digital image for future Umgebung zur Generierung location based services zukünftiger Location Based Services

\section{Bordnetz 2025 / Vehicle Wiring Systems 2025}
U. Siebel,
VOBES NG - Das neue
VOBES NG - Next
R. Gemmerich, Bordnetzentwicklungssystem
D. Peters, im Volkswagen Konzern generation of the Volks-
R. Milke wagen harness development system 

C. Bohne,
Trends shaping the
Trends shaping the
A. Hörtling,
future E/E architecture -
future E/E architecture -
T. Huck, Quo Vadis - Domain and/
Quo Vadis - Domain and/
I. Deak, or zone-oriented E/E
or zone-oriented E/E
O. Koller,
architecture pattern
architecture pattern
K. Hofmann,
A. Lock

55

\section{End to End E/E Architektur / End-to-End E/E Architecture}
R. Zöller,
R. Roppel

R. Grave,

A. Much

B. Gross,
P. Bartsch

T. Scharnhorst,

S. Fürst,

S. Rathgeber,

L. Slansky,

F. Kirschke-Biller,

R. Flores,

T. Jaux,

T. Rüping,

K. Nishikawa,

C. Krömke
Seamless Electronics for

Automotive Services -

Enabler der End-to-End

Elektronikarchitektur der

Zukunft

Neue Kommunikations-

mechanismen für Zentral-

rechner mit Fail Operational

Anforderung

Moderner Ansatz für eine

Gastronomiearchitektur im

Fahrzeug

AUTOSAR als DIE Software

Plattform für intelligente

Mobilität

Seamless electronics for automotive services Enabler of the end-to-end electronics architecture of the future

Communication

mechanism for

fail-operational high

performance controller

Modern approach to in-vehicle infotainment architecture

AUTOSAR proofs to be THE automotive software platform for intelligent mobility 
K. Michels,

T. Drevensek

W. Braunstorfer,

E. Liepold,

A. Gegg
Neue integrierte Plattform

als Antwort auf aktuelle

Anforderungen an die

E/E Architektur platform for EE car

architectures

hardwareunabhängigen hardware agnostic high-

Hochintegrationsfunktion - level function -

Implementierung einer

Implementing a state-of-

hochmodernen

the-art AC control

Klimaregelung

\section{Lichttechnik / Lighting Technology}

F. Langkabel,

T. Kanning

J. Roslak,

C. Wilks,

B. Kubitza

M. Austerer

T. Liebetrau,

S. Grötsch
OPELs IntelliLux LED ${ }^{\circledR}$

Matrix Beleuchtung im

neuen Insignia

Hochauflösende Schein-

werfer: Herausforderungen

für Elektronikarchitekturen

Verbesserung der Fahrsicherheit und des Komforts durch intelligente

Beleuchtung - Elektronik und Networking bereiten den

Weg für neue Use Cases

Auf dem Weg zum intelligenten Scheinwerfer Konzepte für hochaufgelöste Lichtquellen
OPELs IntelliLux LED ${ }^{\circledR}$

Matrix Lights in the new Insignia

High resolution head-

71

lamps: Challenges for

electronic architectures

Intelligent lighting

73

improves driving safety

and comfort - Electronics

and networking pave the

way for new use cases

On the path towards

75 intelligent headlights -

Concepts for high-

resolution light sources 


\section{Security}

Y. Diskin

Intelligence-driven offensive- Intelligence-driven offen-

Defense (I-D O-D) -

A new methodology for

cyber security

S. Römmele

M. Wolf,

R. Lambert
Datensicherheit / Software

Over-The-Air Updates

Ransomware gegen

moderne Fahrzeuge -

Mögliche Angriffspfade und

effektive Schutzmaßnahmen
sive-Defense (I-D O-D) -

A new methodology for

cyber security

(Cyber) Security / over-

79

the-air software updates

Ransomware against

81 modern vehicles -

Feasible attack paths and

effective protection

measures

\section{E-Mobilität 2025 / E-Mobility 2025}

F. Grill

K.-M. Fritsch,

C. Schmuelling,

T. Binder,

M. Cramme

M. Kübel
Der Weg zu einem globalen

Ladestandard -

Harmonisierung der

Ladeschnittstelle

Herausforderungen eines

$48 \mathrm{~V}$-Fahrantriebs mit

$20 \mathrm{~kW}$ mechanischer

Dauerleistung

High Power Charging (CCS) - Hochleitsungsladen und aktuelle Aktivitäten in der internationalen

Standardisierung
The path to a global EV charging system - How to harmonize the customer interface

Challenges of a $48 \mathrm{~V}$ drive system with $20 \mathrm{~kW}$ continuous mechanical power

Combined Charging
System (CCS) -

High Power Charging and

current activities in

international

standardization 

F. Frank,
J. Wilhelm,
D. Schramm

B. Frieß,

T. Soczka-Guth,

F. Hofbeck,

F. Nietfeld

\section{Methoden + Test}

P. Oel,

F. Pohl,

J. Timpner,

B. Aschoff

M. Giertzsch

R. Straschill,

T. Schäfer

P. Ginal,

H. Dörr
SoC-Swing basierte

Optimierung von Fahrzeugbatterien unter Verwendung von Simulationen

Herausforderungen in der

Batterieentwicklung:

Der ausgewogene Mix
SoC-Swing based

optimization of

automotive batteries

using simulations

Challenges in battery

development: The best

fit

89

91
Digital Readiness - Virtuelle Digital readiness - Virtual Funktionsintegration und integration and validation -absicherung im Simulations- in Volkswagen Lab (SimLAB) von Volkswagen

Softwaretestmethoden bei Opel - Nahtloses Testen vom C-Code hin zu Endnutzer-Anforderungen

Anwendung etablierter Methoden zur Quantifizierung von Testabdeckung zur Sicherstellung der Sicherheit selbstlernender Systeme

Testautomatisierung im praktischen Einsatz bei Valeo Siemens eAutomotive Siemens eAutomotive in practice
93 development's Sim $\angle A B$

Software test methods being used at Opel Seamless testing from C-Code to high level feature requirements

Accepting uncertainty Applying proven methods for quantifying test results and test coverage to ensure safety compliance of self-learning systems

Test automation at Valeo 99 

B. Hermann,
Interaktive Spezifizierung
Interactive specification
K. Krumbiegel, und Validierung and validation of highly
M. Richter-Friedel,
hochautomatisierter Fahr-
automated driving
B. Schonlau funktionen in einer Mixed-
Reality-Umgebung
functions in a mixed
reality environment
F. Akkaya,
PST - Powertrain System
PST - Powertrain system
W. Klos, Test: Ein neues Testelement
T. Schwämmle, einer ganzheitlichen
G. Haffke, Erprobungsstrategie für test: A new test element in H.-C. Reuss hybride Fahrzeugantriebe im a holistic test strategy for hybrid vehicle powertrains Produktentwicklungsprozess withinthe product
development process

101

103

UX
A. Jachens,
AR Head-up Display -
M. Zühlsdorf, System Erfordernisse und T. A. Kern, Lösungen zur präzisen H. Abel Augmentierung von Informationen in den Straßenhintergrund

Augmented reality head105 up display - System requirements and solutions concerning precise augmentation of information within the road scene

1. Othersen, Kognitive Übernahmefähig-

Cognitive driver take-over 107

I. Petermann-Stock, keit nach einer pilotierten N. Schömig, Fahrt - MethodenA. Neukum, entwicklung und WechselT. Fuest wirkung mit der capability after piloted driving - method development and interaction with a side task Durchführung von Nebentätigkeiten
A. Patil,
User-centric HMI
User-centric HMI
S. Shinde considering behavioral aspect of driver considering behavioral aspect of driver 
https://doi.org/10.51202/9783181022993-1

Generiert durch IP '172.22.53.54', am 26.04.2023, 10:38:12.

Das Erstellen und Weitergeben von Kopien dieses PDFs ist nicht zulässig. 